Bull. Austral. Math. Soc.

81R05, 20c35, 22E70

Vol. 49 (1994) [177-204]

\title{
QUANTUM DOUBLE FINITE GROUP ALGEBRAS AND LINK POLYNOMIALS
}

\author{
I. Tsohantus and M.D. Gould
}

\begin{abstract}
Unitary representations of the braid group and corresponding link polynomials are constructed corresponding to each irreducible representation of a quantum double finite group algebra. Moreover the diagonal form of the braid generator is derived from which a general closed formula is obtained for link polynomials. As an example, link polynomials corresponding to certain induced representations of the symmetric group and its subgroups are determined explicitly.
\end{abstract}

\section{INTRODUCTION}

A central problem in the theory of knots is the classification of topologically equivalent links [13]. This task is facilitated through the introduction of link polynomials which determine a topological invariant for knots. This approach dates back to the pioneering work of Alexander [2] and Markov [16]. An alternative, but completely compatible, geometric approach was also developed by Reidemeister [17] based on the application of certain operations on links called Reidemeister moves.

The recent work of Jones [12] has revealed a remarkable connection between knot theory and exactly solvable models in statistical mechanics [3, 14]. This connection arises through the Yang-Baxter equation. Solutions to this equation give rise to exactly solvable lattice models and at the same stroke afford a representation, in the spectral parameter limit, of the braid group from which link polynomials can be obtained. A systematic procedure for obtaining solutions to the Yang-Baxter equation came with the introduction of quasi-triangular Hopf algebras [4], and quantum groups in particular $[4,11]$.

It is now generally recognised $[18,20,21]$ that one can obtain a representation of the braid group and corresponding link polynomial from each irreducible representation of a quasi-triangular Hopf algebra. The representation theory and applications of quantum groups in particular have been extensively studied recently $[4,11,18,21$, $6,7]$. Here we investigate link polynomials arising from quantum double finite group algebras and their representations.

Received 30th March, 1993

The authors gratefully acknowledge the financial support of an ARC research grant.

Copyright Clearance Centre, Inc. Serial-fee code: 0004-9729/94 \$A2.00+0.00. 
Corresponding to any finite group $G$ we obtain, through the quantum double construction of Drinfeld [4], a quasi-triangular Hopf algebra of dimension $|G|^{2}$ called the quantum double of $G$ and denoted $D(G)$. This paper is a continuation of a previous investigation [8], herein referred to as $I$, in which the irreducible (unitary) representations of $D(G)$ and corresponding characters were obtained. Below we apply these results to the explicit construction of link polynomials arising from certain induced representations of a finite group.

Unlike the case for quantum groups, representations of the braid group constructed in this way are shown to be unitary. Moreover, it is demonstrated that the braid generator can always be diagonalised on $V \otimes V, V$ an arbitrary irreducible $D(G)$ module, and a generalisation of the Reshetikhin form of the braid generator, previously derived for quantum groups $[18,10]$, is obtained. As an application, a general closed formula is determined for link polynomials.

The structure of the paper is as follows. Section 2 gives a brief introduction to some aspects of the braid group. This is followed up in Section 3 with an outline of the role of quasi-triangular Hopf algebras in the construction of braid group representations and link polynomials. In Section 4 quantum double finite group algebras $D(G)$ are introduced and a brief summary of the results from $I$ is given. In Section 5 we consider link polynomials arising from the irreducible representations of $D(G)$ : in particular the diagonalisability of the braid generator is established. We conclude in Section 6 with an explicit example arising from the symmetric groups $S_{N}(N \geqslant 3)$ and their subgroups.

\section{Braid Group and Link Polynomials}

We begin with the definition of an $n$-braid which is constructed as follows. Consider two rows of $n$ points, numbered $1, \ldots, n$, so that the $i$ th point of the first row is directly above the $i$ th point of the second. Then connect each point of the upper row with one and only one point of the lower one allowing over and under crossings amongst the connecting strings. The configuration so obtained is called an $n$-braid (see Figure 1 ). The trivial $n$-braid is the configuration in which the $i$ th point of the upper row is connected with the $i$ th point of the lower one, for all $i=1, \ldots, n$. By convention, we number the strings with the same number as the one assigned to their end points on the upper row.

We introduce an elementary operation on $n$-braids which consists of making an intersection of the $i$ th string with the $(i+1)$ st such that the $i$ th string passes over the $(i+1)$ st. Obviously there are $n-1$ such operations and to each one of them we assign a generator $\sigma_{i}, 1 \leqslant i<n$ (see Figure 2a). To the inverse operations we assign the inverse generators $\sigma_{i}^{-1}, 1 \leqslant i<n$ (see Figure 2b). Any configuration can be obtained from the trivial braid by successive applications of these operations and can thus be 


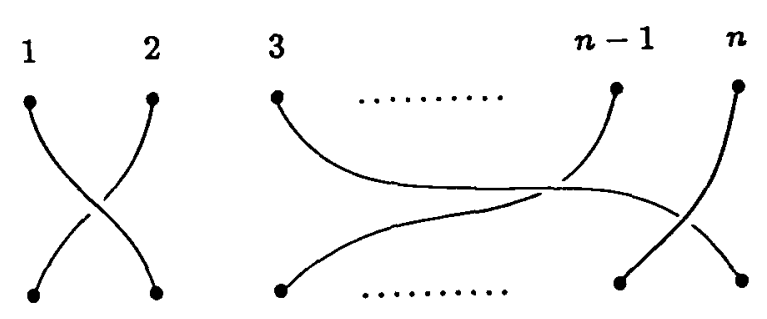

Figure 1

represented as a word in the generators $\sigma_{i}^{ \pm 1}$.

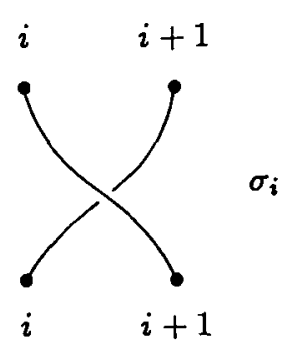

Figure 2a

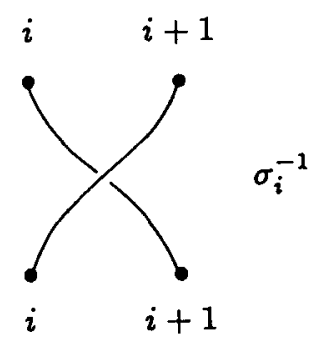

Figure 2b

The $n-1$ generators $\sigma_{i}$ and their inverses generate an infinite discrete group called the braid group, denoted $B_{n}$, with identity given by the trivial braid. It is crucial to observe that in general the expression of a braid configuration in terms of a word $\theta$ is not unique. We thus endow the braid group $B_{n}$ with additional relations between the generators $\sigma_{i}$, which guarantee the equivalence between different words representing the same braid configuration. These relations read as follows:

$$
\begin{aligned}
\sigma_{i} \sigma_{j} & =\sigma_{j} \sigma_{i}, & & |i-j| \geqslant 2 \\
\sigma_{i} \sigma_{i+1} \sigma_{i} & =\sigma_{i+1} \sigma_{i} \sigma_{i+1}, & & 1 \leqslant i<n-1
\end{aligned}
$$

and are considered as the defining relations of the braid group $B_{n}$.

For each $\theta \in B_{n}$ we let $\hat{\theta}$ denote the closure of the braid represented by $\theta$ : obtained by joining opposite ends of the braid together. The diagram so obtained is called a link diagram and may be considered as the projection (or shadow) of a link onto the plane. Any closed braid represents a link and by Alexander's theorem [2] every link is represented by a closed braid. It is important to note however, that the representation of a link as a closed braid is not unique. 
The problem of non-uniqueness of this representation was resolved by Markov [16] who defined two types of operations amongst braids giving the same link, which transform one braid into the other. These operations are:

$$
\begin{aligned}
\text { (I) } \quad \theta \eta & \rightarrow \eta \theta, & & \theta, \eta \in B_{n} \\
\text { (II) } \quad \theta & \rightarrow \theta \sigma_{n-1}^{ \pm 1}, & & \theta \in B_{n-1} \subset B_{n}
\end{aligned}
$$

and are called Markov moves.

The process of classifying topologically equivalent (or ambient isotopic) links, without the use of the braid group, can be highly tedious. The original approach of Reidemeister [17] involves the so-called Reidemeister moves which transform equivalent links into each other by continuously deforming the link diagram in a particular way without tearing the strings. The introduction of the braid group affords an elegant algebraic method of constructing topological invariants which facilitate the classification of equivalent links.

A central role in the construction of such invariants is played by the Markov trace which is defined as a mapping $\varphi: B_{n} \rightarrow \mathbb{C}$ with the following properties:

$$
\begin{array}{ll}
\varphi(\theta \eta)=\varphi(\eta \theta), & \forall \theta, \eta \in B_{n} \\
\varphi\left(\theta \sigma_{n-1}\right)=z \varphi(\theta), & \forall \theta \in B_{n-1} \subset B_{n} \\
\varphi\left(\theta \sigma_{n-1}^{-1}\right)=\bar{z} \varphi(\theta), & \forall \theta \in B_{n-1} \subset B_{n}
\end{array}
$$

where

$$
z=\varphi\left(\sigma_{n-1}\right), \quad \bar{z}=\varphi\left(\sigma_{n-1}^{-1}\right) \text {. }
$$

A link polynomial $L(\widehat{\theta}), \theta \in B_{n}$, may then be constructed by setting

$$
L(\widehat{\theta})=(z \bar{z})^{-(n-1) / 2}\left(\frac{\bar{z}}{z}\right)^{e(\theta) / 2} \varphi(\theta), \quad \forall \theta \in B_{n}
$$

where $e(\theta)$ is the sum of the exponents of the $\sigma_{i}^{\prime}$ s appearing in the word $\theta . L(\hat{\theta})$ is clearly a topological invariant for links since it satisfies the following relations

$$
\begin{aligned}
I(\widehat{\theta \eta}) & =L(\widehat{\eta \theta}), \quad \forall \theta, \eta \in B_{n} \\
L\left(\widehat{\theta \sigma}_{n-1}^{ \pm 1}\right) & =L(\widehat{\theta}), \quad \forall \theta \in B_{n-1} \subset B_{n}
\end{aligned}
$$

which are a direct consequence of the properties (2) of the Markov trace.

Well known examples of such link polynomials are afforded by the Alexander [2] and Jones [12] polynomials. A large class of link polynomials is now known mainly as a result of the successful attempts to construct representations of the braid group and 
corresponding Markov traces from exactly solvable models [14] and representations of quantum groups and supergroups $[18,20,21]$. We now briefly outline the procedure for constructing link polynomials corresponding to any finite dimensional irreducible representation of a quasi-triangular Hopf algebra. We then focus attention on the important special case of quantum double finite group algebras.

\section{QUaSi-TRIangUlar HoPf algebras AND LINK POLYNOMIALS}

Let $A$ be a Hopf algebra over $\mathbb{C}$, with identity $1 \in A$, coproduct $\triangle: A \rightarrow A \otimes A$, counit $\varepsilon: A \rightarrow \mathbb{C}$ and bijective antipode $S: A \rightarrow A$. For the full defining relations of a Hopf algebra we refer the reader to $[19,1]$. Following Sweedler's sigma notation [19], we write

$$
\triangle(a)=\sum_{(a)} a^{(1)} \otimes a^{(2)}, \quad \forall a \in A
$$

in terms of which the counit and antipode properties are expressible

$$
\begin{aligned}
a & =\sum_{(a)} a^{(1)} \varepsilon\left(a^{(2)}\right)=\sum_{(a)} \varepsilon\left(a^{(1)}\right) a^{(2)} \\
\varepsilon(a)= & \sum_{(a)} a^{(1)} S\left(a^{(2)}\right)=\sum_{(a)} S\left(a^{(1)}\right) a^{(2)} .
\end{aligned}
$$

Next we introduce the twist map $T: A \otimes A \rightarrow A \otimes A$ defined by

$$
T(a \otimes b)=b \otimes a, \quad \forall a, b \in A .
$$

Then it is known [19] that $A$ also constitutes a Hopf algebra under the opposite coproduct $\Delta^{T}=T \cdot \Delta$ with antipode $S^{-1}$ and counit $\varepsilon$. In the case that $\Delta^{T}=\Delta$ we call $A$ cocommutative: it can be shown [19] that if $A$ is commutative or cocommutative then $S^{2}=I$, the identity map on $A$. Following Drinfeld [4] we can now define a quasi-triangular Hopf algebra as follows:

Definition 3.1: A Hopf algebra $A$ is called quasi-triangular if there exists an invertible element

$$
R=\sum_{i} a_{i} \otimes b_{i} \in A \otimes A
$$

satisfying

and

$$
\begin{gathered}
\triangle^{T}(a) R=R \Delta(a), \quad \forall a \in A \\
(\Delta \otimes I) R=R_{12} R_{13}, \quad(I \otimes \Delta) R=R_{13} R_{12}
\end{gathered}
$$

where we have adopted the usual convention

$$
R_{12}=\sum_{i} a_{i} \otimes b_{i} \otimes 1, \quad R_{13}=\sum_{i} a_{i} \otimes 1 \otimes b_{i} \quad \text { et cetera. }
$$


Remarks. If $A$ is a quasi-triangular Hopf algebra with canonical element $R$, then $R^{T}=T \cdot R$ is easily seen to satisfy

$$
\begin{gathered}
\triangle(a) R^{T}=R^{T} \triangle^{T}(a), \quad \forall a \in A \\
\left(\triangle^{T} \otimes I\right) R^{T}=R_{12}^{T} R_{13}^{T}, \quad\left(I \otimes \triangle^{T}\right) R^{T}=R_{13}^{T} R_{12}^{T}
\end{gathered}
$$

and

so that $A$ also constitutes a quasi-triangular Hopf algebra under the opposite coproduct $\Delta^{T}$ with canonical element $R^{T}$.

A direct consequence of the above definition is that the canonical element $R$, called the universal $R$-matrix, satisfies the Yang-Baxter equation

$$
R_{12} R_{13} R_{23}=R_{23} R_{13} R_{12}
$$

of importance in the theory of exactly solvable models in statistical mechanics [3, 14]. Thus, corresponding to each irreducible $A$-module $V$, a solution to the Yang-Baxter equation on $V \otimes V \otimes V$ is thereby obtained. As seen below, equation (8) is also responsible for the connection with the braid group and link polynomials.

A large class of such algebras is afforded by the quantum double construction of Drinfeld $[4,8]$ whereby a quasi-triangular Hopf algebra is manufactured (under certain mild conditions) from any Hopf algebra and its dual. Important examples of quantum double Hopf algebras are afforded by quantum groups, which have been extensively studied recently $[4,11,18,21,6,7]$, and quantum double finite group algebras [8]. The representation theory of these latter algebras was investigated in [8] where the universal $R$-matrix was also determined in fully explicit form. Below we utilise these results to obtain link polynomials arising from certain induced representations of a finite group.

Returning to the general case, let $A$ be a quasi-triangular Hopf algebra with canonical element $R$. Following Drinfeld [5], if $R$, as given by equation (5), has inverse

$$
R^{-1}=\sum_{i} c_{i} \otimes d_{i} \in A \otimes A
$$

then it can be shown that

$$
u=\sum_{i} S\left(b_{i}\right) a_{i}
$$

has inverse

$$
u^{-1}=\sum_{i} S^{-1}\left(d_{i}\right) c_{i}
$$

and satisfies 


$$
\begin{aligned}
S^{2}(a) & =u a u^{-1}, \quad \forall a \in A \\
\triangle(u) & =(u \otimes u)\left(R^{T} R\right)^{-1} .
\end{aligned}
$$

The element $u$ plays a crucial role in the construction of invariant bilinear forms and central elements [10] for $A$.

Throughout, given a finite dimensional irreducible $A$-module $V_{\Lambda}$, we let $\pi_{\Lambda}$ be the representation afforded by $V_{\Lambda}$ and set

$$
d_{u}[\Lambda]=\operatorname{tr} \pi_{\Lambda}(u)
$$

herein called the $u$-dimension of $V_{\Lambda}$. Recall that the centre $C$ of $A$ is given by

$$
C=\{c \in A \mid c a=a c, \quad \forall a \in A\} .
$$

The role of the element $u$ in the construction of central elements is revealed in the following result proved in [10]:

THEOREM 3.1. If $\omega \in A \otimes$ End $V_{\Lambda}$ satisfies

$$
\left(I \otimes \pi_{\lambda}\right) \triangle(a) \omega=\omega\left(I \otimes \pi_{\Lambda}\right) \triangle(a), \quad \forall a \in A
$$

with $I$ the identity map on $A$, then

$$
c=I \otimes \operatorname{tr}\left\{\left[1 \otimes \pi_{\Lambda}(u)\right] \omega\right\}
$$

belongs to the centre of $A$. Moreover the eigenvalue $\langle c\rangle_{\mu}$ of $c$ on an irreducible module $V_{\mu}$ is given by

$$
d_{u}[\mu](c\rangle_{\mu}=\operatorname{tr}_{V_{\mu} \otimes V_{\Lambda}}[(u \otimes u) \omega] .
$$

Corollary. The elements

$$
c_{m}^{\Lambda}=I \otimes \operatorname{tr}\left\{\left[1 \otimes \pi_{\Lambda}(u)\right]\left(R^{T} R\right)^{m}\right\}
$$

belong to the centre of $A$.

Proof: In view of equations $(6,7)$ the elements

$$
\omega_{m}^{\Lambda}=\left(I \otimes \pi_{\Lambda}\right)\left(R^{T} R\right)^{m}, \quad m \in \mathbb{Z}
$$

satisfy the condition of the theorem from which it follows that

$$
c_{m}^{\Lambda}=I \otimes \operatorname{tr}\left\{\left(1 \otimes \pi_{\Lambda}(u)\right) \omega_{m}^{\Lambda}\right\}
$$

are central elements. 
REMARKS. The proof of Theorem 3.1 is a direct consequence of equation $\left(10_{a}\right)$ satisfied by $u$. This relation clearly determines $u$ uniquely modulo the set of invertible central elements, herein denoted $C_{I}$. Note that $S\left(u^{-1}\right)$ satisfies $\left(10_{a}\right)$ and thus $u S(u)$ is an invertible central element. Any element of $C_{I} u$ may be used in place of $u$ for the construction of central elements. In particular if $S^{2}=I$, the identity map on $A$, as is the case for quantum double finite group algebras, it is convenient to take the identity element $1 \in A$ in place of $u$.

To see the connection with the braid group let $V=V_{\Lambda}$ be a finite dimensional irreducible $A$-module with non-zero $u$-dimension. Let $P$ be the permutation operator on $V \otimes V$ defined by

$$
P v \otimes w=w \otimes v, \quad \forall v, w \in V
$$

and set

$$
\sigma=P R
$$

Here and below we regard elements of $A$ as operators on $V$.

Then equation (6) implies

$$
\sigma \Delta(a)=\Delta(a) \sigma, \quad \forall a \in A
$$

and the Yang-Baxter equation (8) satisfied by $R$ on $V \otimes V \otimes V$ may be written $[4,11$, $18,20,21]$

$$
(I \otimes \sigma)(\sigma \otimes I)(I \otimes \sigma)=(\sigma \otimes I)(I \otimes \sigma)(\sigma \otimes I)
$$

Equation $\left(13_{a}\right)$ states that $\sigma$ and its inverse $\sigma^{-1}=R^{-1} P$ are invariants acting on $V \otimes V$. The next lemma then follows from Theorem 3.1.

LEMMA 3.1. The operators

$$
c=\tau_{2}[(1 \otimes u) \sigma], \quad \bar{c}=\tau_{2}\left[(1 \otimes u) \sigma^{-1}\right],
$$

where $\tau_{2}$ denotes the partial trace taken over the second factor of the tensor product space, are $A$-invariants [10] acting on $V$ with eigenvalues given by

$$
\begin{aligned}
& \gamma_{\Lambda}=\operatorname{tr} V \otimes V[(u \otimes u) \sigma] / d_{u}[\Lambda] \\
& \bar{\gamma}_{\Lambda}=\operatorname{tr} V \otimes V\left[(u \otimes u) \sigma^{-1}\right] / d_{u}[\Lambda]
\end{aligned}
$$

respectively.

Turning our attention to the $n$ th-rank tensor product space

$$
V^{n} \equiv V \otimes V \otimes \ldots \otimes V, \quad(n \text { factors })
$$


equation $\left(13_{b}\right)$ implies that the operators $\sigma_{i}, \sigma_{i}^{-1} \in \operatorname{End}\left(V^{n}\right)$ defined by

$$
\sigma_{i}^{ \pm 1}=\underbrace{I \otimes \ldots \otimes I}_{(i-1)} \otimes \sigma \otimes \underbrace{I \otimes \ldots \otimes I}_{(n-i-1)}, \quad 1 \leqslant i<n
$$

satisfy the defining relations (1) of the braid group and thus give rise to a representation of $B_{n}$ : throughout, for notational simplicity, we identify elements of $B_{n}$ with their (matrix) representatives on $V^{n}$. We are now in a position to construct a Markov trace.

Theorem 3.2. $\varphi: B_{n} \rightarrow \mathbb{C}$, defined by

$$
\varphi(\theta)=\operatorname{tr}\left(u^{\otimes n} \theta\right) / \operatorname{tr}\left(u^{\otimes n}\right), \quad \theta \in B_{n},
$$

where the trace is taken over the $n$-fold tensor product space, satisfies the defining relations (2) of a Markov trace with

$$
z=\gamma_{\Lambda} / d_{u}[\Lambda], \quad \bar{z}=\bar{\gamma}_{\Lambda} / d_{u}[\Lambda] .
$$

Proof: By definition, on $V \otimes V$ we have

$$
\sigma^{2}=R^{T} R
$$

and hence, from equation $\left(10_{b}\right)$,

$$
u \otimes u=\Delta(u) \sigma^{2}=\sigma^{2} \Delta(u) .
$$

It follows that $u \otimes u$ commutes with $\sigma$ and hence $u^{\otimes n}$ must commute with the elements of $B_{n}$ acting on $V^{n}$. From the properties of trace it follows immediately that

$$
\varphi(\theta \eta)=\varphi(\eta \theta), \quad \forall \theta, \eta \in B_{n}
$$

which is property (I) of the Markov trace.

As to property (II) we have, for any $\theta \in B_{n-1} \subset B_{n}$,

$$
\varphi\left(\theta \sigma_{n-1}\right)=t r_{n-1}\left[\tau_{n}\left(u^{\otimes n} \theta \sigma_{n-1}\right)\right] / t r_{n-1}\left[\tau_{n}\left(u^{\otimes n}\right)\right]
$$

where $t r_{n-1}$ represents the trace taken over the first $(n-1)$ factors of the tensor product space $V^{n}$ and $\tau_{n}$ is the partial trace taken over the last factor space. Now

$$
\tau_{n}\left(u^{\otimes n}\right)=d_{u}[\Lambda] u^{\otimes(n-1)}
$$

and, using Lemma 3.1 ,

$$
\begin{aligned}
\tau_{n}\left(u^{\otimes n} \theta \sigma_{n-1}\right) & =u^{\otimes(n-1)} \theta \tau_{2}[(1 \otimes u) \sigma] \\
& =\gamma_{\Lambda} u^{\otimes(n-1)} \theta .
\end{aligned}
$$


Hence substituting into equation $\left({ }^{*}\right)$ we obtain

$\phi\left(\theta \sigma_{n-1}\right)=z \phi(\theta)$
and similarly $\quad \phi\left(\theta \sigma_{n-1}^{-1}\right)=\bar{z} \phi(\theta)$

with $z, \bar{z}$ as in equation (17).

Utilising the above Markov trace and equation (3), we immediately arrive at:

THEOREM 3.3 .

$$
L(\widehat{\theta})=\left(d_{u}[\Lambda]\right)^{n-1}\left(\gamma_{\Lambda} \bar{\gamma}_{\Lambda}\right)^{-(n-1) / 2}\left(\frac{\bar{\gamma}_{\Lambda}}{\gamma_{\Lambda}}\right)^{-e(\theta) / 2} \varphi(\theta), \theta \in B_{n}
$$

where $e(\theta)$ is the sum of the exponents of the $\sigma_{i}$ 's appearing in $\theta$, defines a link polynomial.

In this way link polynomials may be obtained corresponding to any finite dimensional irreducible representation of a quasi-triangular Hopf algebra. Below we investigate in detail link polynomials arising from quantum double finite group algebras.

\section{QUANTUM DOUBLE FINITE GROUP ALGEBRAS}

In this section we briefly summarise the results of paper I: we employ the same notation and conventions throughout. Let $A$ denote the group algebra of a finite group $G$ over the complex field $\mathbb{C}$. Then $A$ becomes a co-commutative Hopf algebra with coproduct, antipode and counit respectively defined by

$$
\triangle(g)=g \otimes g, \quad S(g)=g^{-1}, \quad \varepsilon(g)=1, \quad \forall g \in G
$$

which we extend linearly to all of $A$ in an obvious way.

The dual space $A^{*}$ has a basis of elements $g^{*}, g \in G$, defined by

$$
\left\langle g^{*}, h\right\rangle=\delta(g, h), \quad \forall h \in G
$$

and becomes a commutative algebra with product

$$
g^{*} h^{*}=\delta(g, h) g^{*}, \quad \forall g, h \in G .
$$

$A^{*}$ then inherits the structure of a Hopf algebra from that of $A$ with coproduct $\Delta_{0}$, antipode $S_{0}$ and counit $\varepsilon_{0}$ given respectively by [8]

$$
\begin{gathered}
\triangle_{0}\left(g^{*}\right)=\sum_{h \in G}\left(h^{-1} g\right)^{*} \otimes h^{*}=\sum_{h \in G} h^{*} \otimes\left(g h^{-1}\right)^{*} \\
S_{0}\left(g^{*}\right)=\left(g^{-1}\right)^{*}, \varepsilon_{0}\left(g^{*}\right)=\delta(g, 1), \quad \forall g \in G
\end{gathered}
$$


where 1 denotes the identity element of $G$ and $A$. We note that the identity of $A^{*}$ is given by the counit $\varepsilon$ on $A$ which is expressible

$$
\varepsilon=\sum_{g \in G} g^{*}
$$

In view of equation (21) we have

$$
\triangle_{0}(\varepsilon)=\varepsilon \otimes \varepsilon, \quad S_{0}(\varepsilon)=\varepsilon, \quad \varepsilon_{0}(\varepsilon)=1 .
$$

We may now introduce the quantum double $D(G)$ of the group algebra $A$ which is the $|G|^{2}$-dimensional vector space spanned by all free products

$$
g h^{*}, \quad g, h \in G
$$

which becomes an associative algebra with the definition

$$
h^{*} g=g\left(g^{-1} h g\right)^{*} .
$$

Following the quantum double construction $[4,8], D(G)$ then becomes a quasitriangular Hopf algebra with coproduct $\bar{\Delta}$, antipode $\bar{S}$ and counit $\bar{\varepsilon}$ given respectively by

$$
\begin{aligned}
& \bar{\triangle}\left(g h^{*}\right)=\Delta(g) \triangle_{0}\left(h^{*}\right)=\sum_{k \in G} g\left(k^{-1} h\right)^{*} \otimes g k^{*} \\
& \bar{S}\left(g h^{*}\right)=S_{0}\left(h^{*}\right) S(g)=\left(h^{-1}\right)^{*} g^{-1}=g^{-1}\left(g h^{-1} g^{-1}\right)^{*} \\
& \bar{\varepsilon}\left(g h^{*}\right)=\varepsilon(g) \varepsilon_{0}\left(h^{*}\right)=\delta(h, 1), \quad \forall g, h \in G .
\end{aligned}
$$

The corresponding canonical element $R$ is given by [8]

$$
R=\sum_{g \in G} g \otimes g^{*}
$$

which necessarily satisfies the Yang-Baxter equation (8).

It can be shown [4] that the $R$-matrix (23a) has inverse

$$
R^{-1}=(\bar{S} \otimes I) R=\sum_{g \in G} g^{-1} \otimes g^{*},
$$

as may be verified directly. As in $I$, we here regard $A$ and $A^{*}$ as subalgebras of $D(G)$ by identifying $g \cdot \varepsilon$ and $1 \cdot g^{*}$ with $g, g^{*}$ respectively, for all $g \in G$. We note that the antipode $\bar{S}$ satisfies

$$
\bar{S}^{2}=I
$$


I the identity map on $D(G)$, so that $D(G)$ is an involutory Hopf algebra [15]. The identity element of $D(G)$ is given by $1 \cdot \varepsilon$ which we identify with $1 \in G$.

We now say something about the representation theory of $D(G)$. Following $I$, we have:

Definition 4.1: A finite dimensional $D(G)$ module $V$ is called unitary if it can be equipped with an inner product $($,$) such that for all g, h \in D(G)$

$$
\left(g h^{*} v, w\right)=\left(v, h^{*} g^{-1} w\right), \quad \forall v, w \in V .
$$

Equivalently, if $\pi$ is the representation of $D(G)$ afforded by $V$, then $V$ is called unitary if it can be equipped with an inner product such that

$$
\pi\left(h^{*} g\right)^{\dagger}=\pi\left(g^{-1} h^{*}\right)
$$

where $\dagger$ denotes Hermitian conjugate. The following result was proved in $I$ :

LEMMA 4.1. Every finite dimensional $D(G)$ module is equivalent to a unitary one.

It follows, from standard arguments, that every finite dimensional $D(G)$ module is completely reducible. In particular we have [8]:

THEOREM 4.1. $D(G)$ is a semi-simple of algebra.

The irreducible $D(G)$-modules were classified in $I$ and may be constructed as follows. Let $\mathcal{C}_{k}(1 \leqslant k \leqslant n)$ denote the conjugacy classes of $G$ with $\mathcal{C}_{1}=\{1\}$ the conjugacy class of the identity $1 \in G$. For each $k=1, \ldots, n$ choose a fixed conjugacy class representative $g_{k} \in \mathcal{C}_{k}$ and let $Z_{k}$ be the centraliser subgroup of $g_{k}$; namely

$$
Z_{k}=\left\{h \in G \mid h g_{k}=g_{k} h\right\} \text {. }
$$

We denote the group algebra of $Z_{k}$ by $A_{k}$ : then [8]

$$
\operatorname{dim} A_{k}=\left|Z_{k}\right|=|G| /\left|\mathcal{C}_{k}\right|
$$

For $s \in \mathcal{C}_{k}$ we choose a fixed $\tau_{s} \in G$ such that

$$
s=\tau_{\imath} g_{k} \tau_{e}^{-1}
$$

for simplicity when $s=g_{k}$ we take $\tau_{a}=1$. Given an irreducible $A_{k}$-module $V_{\alpha}^{k}$ we have the corresponding induced $A$-module

$$
V_{k, \alpha}=A \otimes_{A_{k}} V_{\alpha}^{k}
$$

which is spanned by vectors

$$
v(s)=\tau_{a} \otimes v, \quad v \in V_{\alpha}^{k}, \quad s \in \mathcal{C}_{k} .
$$


Then $V_{k, \alpha}$ becomes a $D(G)$ module under the action defined by [8]

$$
g h^{*} v(s)=\delta(h, s)\left(\tau_{g s g^{-1}}^{-1} g \tau, v\right)\left(g s g^{-1}\right)
$$

With this construction we have [8]:

THEOREM 4.2. $V_{k, \alpha}$ is an irreducible $D(G)$-module. Moreover every irreducible $D(G)$ module is isomorphic to one of the $V_{k, \alpha}$.

Following $I$, it can be shown that there is in fact a $1-1$ correspondence between the irreducible $D(G)$-modules and the induced modules $V_{k, \alpha}$ as $k$ runs through the conjugacy classes of $G$ and $\alpha$ through the non-isomorphic irreducible $A_{k}$-modules.

It is worth noting that given an inner product $($,$) on V_{\alpha}^{k}$, we have the induced inner product on $V_{k, \alpha}$ defined by

$$
(w(t), v(s))=\delta(t, s)(w, v), \quad \forall v, w \in V_{\alpha}^{k}, \quad s, t \in \mathcal{C}_{k}
$$

and extended by linearity in an obvious way. With this definition it is easily verified that $V_{k, \alpha}$ gives rise to an irreducible unitary $D(G)$ module, provided $V_{\alpha}^{k}$ is assumed unitary for $A_{k}$, in agreement with Lemma 4.1.

Hence, without loss of generality, we assume $V_{k, \alpha}$ gives rise to a unitary $D(G)$ module and we let $\pi_{k, \alpha}$ be the matrix representation afforded by $V_{k, \alpha}$. It follows from equations (23) that the $R$-matrix

$$
\begin{aligned}
R & =\sum_{g \in G} \pi_{k, \alpha}(g) \otimes \pi_{k, \alpha}\left(g^{*}\right) \\
& =\sum_{s \in \mathfrak{c}_{k}} \pi_{k, \alpha}(s) \otimes \pi_{k, \alpha}\left(s^{*}\right)
\end{aligned}
$$

has inverse

$$
R^{-1}=\sum_{, \in \mathcal{C}_{k}} \pi_{k, \pi}\left(s^{-1}\right) \otimes \pi_{k, \alpha}\left(s^{*}\right)
$$

and satisfies the Yang-Baxter equation (8) on $V_{k, \alpha}^{\otimes s}$. We note that equation (25) implies the Hermiticity condition

$$
R^{\dagger}=R^{-1}
$$

so that $R$ is a unitary operator.

To obtain the matrices (28) in more explicit form, introduce an orthonormal basis $\left\{v_{i} \mid 1 \leqslant i \leqslant d_{\alpha}^{k}\right\}$ for the irreducible $A_{k}$-module $V_{\alpha}^{k}$ so that the vectors

$$
v_{i}(s), \quad s \in \mathcal{C}_{k}, \quad 1 \leqslant i \leqslant d_{\alpha}^{k}
$$


give rise to an orthonormal basis for $V_{k, \alpha}$ : here and below, we follow $I$ and set $d_{\alpha}^{k}=$ $\operatorname{dim} V_{\alpha}^{k}$, so that the irreducible $D(G) \operatorname{module} V_{k, \alpha}$ has dimension

$$
d[k, \alpha]=\left|\mathcal{C}_{k}\right| d_{\alpha}^{k}
$$

We let $E_{j t}^{i s}\left(s, t \in \mathcal{C}_{k}\right)$ denote the corresponding elementary matrices with a 1 in the $(i s, j t)$ position and zeros elsewhere.

According to equation (27) the action of $D(G)$ in the basis (30) is given by [8]

$$
g h^{*} v(s)_{i}=\delta(h, s) \pi_{\alpha}^{k}\left(\tau_{g \circ g^{-1}}^{-1} g \tau_{\alpha}\right)_{j i} v\left(g s g^{-1}\right)_{j}
$$

where $\pi_{\alpha}^{k}$ is the representation of $A_{k}$ afforded by $V_{\alpha}^{k}$ : here we have adopted the convention, maintained throughout the paper, of summing over repeated indices. We thus arrive at the following construction for the matrix representation $\pi_{k, \alpha}$ in the basis $(30)$ :

$$
\pi_{k, \alpha}\left(g h^{*}\right)_{j t, i \_}=\delta(h, s) \delta\left(t, g s g^{-1}\right) \pi_{\alpha}^{k}\left(\tau_{t}^{-1} g \tau_{\iota}\right)_{j i}
$$

Substituting equation (31) into equations (28) we arrive at the following explicit construction for the $R$-matrices (28) in terms of elementary matrices [8]:

$$
\begin{aligned}
& R=\sum_{s, t \in \mathcal{C}_{k}} \pi_{\alpha}^{k}\left(\tau_{a t s^{-1}}^{-1} s \tau_{t}\right)_{j i} E_{i t}^{j s t s^{-1}} \otimes E_{l s}^{l}
\end{aligned}
$$

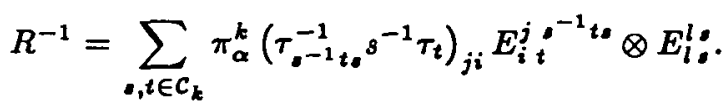

In particular when $\alpha=\iota_{0}$, corresponding to the identity representation of $A_{k}$, equations (32) reduce to the $\left|\mathcal{C}_{k}\right|^{2} \times\left|\mathcal{C}_{k}\right|^{2}$ matrices given by

$$
\begin{aligned}
R & =\sum_{s, t \in \mathcal{C}_{k}} E_{t}^{s t_{s}^{-1}} \otimes E^{\prime} \\
R^{-1} & =\sum_{s, t \in \mathcal{C}_{k}} E_{t}^{s-1} t_{s} \otimes E_{s}^{\prime}
\end{aligned}
$$

Following Section 3, we may use the above $R$-matrix formulae to construct explicitly link polynomials corresponding to each irreducible module $V_{k, \alpha}$. This problem is investigated in detail in Section 5 below. We first require some results on the character theory of $D(G)$ and tensor product decompositions.

We define the character $\chi_{k, \alpha}$, corresponding to the irreducible module $V_{k, \alpha}$, in the usual way; namely

$$
\chi_{k, \alpha}(a)=\operatorname{tr} \pi_{k, \alpha}(a), \quad \forall a \in D(G)
$$


From equation (31), we have immediately

$$
\chi_{k, \alpha}\left(g s^{*}\right)= \begin{cases}0, & s \notin \mathcal{C}_{k} \\ \delta\left(s, g s g^{-1}\right) \chi_{\alpha}^{k}\left(\tau_{s}^{-1} g \tau_{s}\right), & s \in \mathcal{C}_{k}\end{cases}
$$

where $\chi_{\alpha}^{k}$ is the character of the $A_{k}$-module $V_{\alpha}^{k}$. Following $I$, if we denote the centraliser subgroup of $s \in G$ by $Z(s)$, then equation (34) implies

$$
\chi_{k, \alpha}\left(g s^{*}\right)=0, \quad \text { unless } g \in Z(s) .
$$

As for the case of finite groups, we may now construct projection operators onto the irreducible $D(G)$ modules $V_{k, \alpha}$ according to [8]

$$
\begin{aligned}
E_{k, \alpha} & =\frac{d[k, \alpha]}{|G|} \sum_{g, h \in G} \chi_{k, \alpha}\left(g^{-1} h^{*}\right) h^{*} g \\
& =\frac{d[k, \alpha]}{|G|} \sum_{s \in C_{k}} \sum_{g \in Z(\lrcorner)} \chi_{k, \alpha}\left(g^{-1} s^{*}\right) s^{*} g .
\end{aligned}
$$

These operators form an orthogonal set of idempotents, adding up to the identity, and spanning the centre $\bar{C}$ of $D(G)$. This implies, in particular, the following [8]:

THEOREM 4.3. The characters $\chi_{k, \alpha}$ satisfy the first orthogonality relation

$$
\sum_{g, h \in G} \chi_{k, \alpha}\left(g^{-1} h^{*}\right) \chi_{l, \beta}\left(h^{*} g\right)=|G| \delta_{k l} \delta_{\alpha \beta} \text {. }
$$

REMARKS.

(1) It can be shown' [8] that the above characters also satisfy a second orthogonality relation but this will not be required below.

(2) In the special case $(k, \alpha)=\left(1, \iota_{0}\right)$ corresponding to the identity representation of $D(G)$, where $\iota_{0}$ here refers to the identity representation of $G=Z_{1}$, the central idempotent (36) reduces to

$$
E_{1, \iota_{0}}=\frac{1}{|G|} \sum_{g \in G} g 1^{*}
$$

and determines a left integral [19] in $D(G)$.

Finally, for later use we note that given two irreducible modules $V_{k, \alpha}, V_{l, \beta}$ their tensor product obviously becomes a $D(G)$ module under the action determined by the coproduct $\bar{\Delta}$. We write the decomposition of this tensor product module into irreducible submodules as

$$
V_{k, \alpha} \otimes V_{l, \beta}=\bigoplus_{j, \gamma} m_{j, \gamma} V_{j, \gamma}
$$

where $m_{j, r} \in \mathbb{Z}_{+}$denotes the multiplicity of the irreducible module $V_{j, \gamma}$ in the tensor product space. We have: 
LEMMA 4.2 .

$$
m_{j, \gamma}=\frac{1}{\left|Z_{k}\right|} \sum_{s \in \mathcal{C}_{l}} \sum_{g \in Z_{k} \cap Z(s)} \chi_{j, \gamma}\left[g^{-1}\left(s g_{k}\right)^{*}\right] \chi_{k, \alpha}\left(g_{k}^{*} g\right) \chi_{l, \beta}\left(s^{*} g\right) \text {. }
$$

Proof: It follows from Lemma 5.2 of I that

$$
\begin{aligned}
& m_{j, \gamma}=\frac{1}{|G|} \sum_{,, g, t \in G} \chi_{j, \gamma}\left[g^{-1}(s t)^{*}\right] \chi_{k, \alpha}\left(t^{*} g\right) \chi_{l, \beta}\left(s^{*} g\right) \\
& =\frac{1}{|G|} \sum_{\substack{t \in \mathcal{C}_{k} \\
s \in \mathcal{C}_{l}}} \sum_{\substack{(t) \cap) \cap Z(s)}} \chi_{j, \gamma}\left[g^{-1}(s t)^{*}\right] \chi_{k, \alpha}\left(t^{*} g\right) \chi_{l, \beta}\left(s^{*} g\right) \text {. }
\end{aligned}
$$

On the other hand, writing $t=\tau_{t} g_{k} \tau_{t}^{-1}$ and using the invariance properties of characters [8], we have

$$
\begin{aligned}
\chi_{j, \gamma}\left[g^{-1}(s t)^{*}\right] & =\chi_{j, \gamma}\left[\left(\tau_{t}^{-1} g^{-1} \tau_{t}\right)\left(\tau_{t}^{-1} s \tau_{t} g_{k}\right)^{*}\right] \\
\chi_{k, \alpha}\left(t^{*} g\right) & =\chi_{k, \alpha}\left(g_{k}^{*} \tau_{t}^{-1} g \tau_{t}\right) \\
\chi_{l, \beta}\left(s^{*} g\right) & =\chi_{l, \beta}\left[\left(\tau_{t}^{-1} s \tau_{t}\right)^{*}\left(\tau_{t}^{-1} g \tau_{t}\right)\right] .
\end{aligned}
$$

Substituting into the sum above and replacing $s, g$ with $\tau_{t}^{-1} s \tau_{t}, \tau_{t}^{-1} g \tau_{t}$ respectively, we arrive at

$$
\begin{aligned}
m_{j, \gamma} & =\frac{1}{|G|} \sum_{t \in \mathcal{C}_{k}} \sum_{s \in \mathcal{C}_{l}} \sum_{g \in Z_{k} \cap Z(\bullet)} \chi_{j, \gamma}\left[g^{-1}\left(s g_{k}\right)^{*}\right] \chi_{k, \alpha}\left(g_{k}^{*} g\right) \chi_{l, \beta}\left(s^{*} g\right) \\
& =\frac{\left|\mathcal{C}_{k}\right|}{|G|} \sum_{s \in \mathcal{C}_{l}} \sum_{g \in Z_{k} \cap Z(\bullet)} \chi_{j, \gamma}\left[g^{-1}\left(s g_{k}\right)^{*}\right] \chi_{k, \alpha}\left(g_{k}^{*} g\right) \chi_{l, \beta}\left(s^{*} g\right)
\end{aligned}
$$

which is sufficient to prove the result.

REMARK. It is worth noting that $m_{j, \gamma}=0$ unless

$$
\mathcal{C}_{j} \subseteq \mathcal{C}_{l} \cdot \mathcal{C}_{k}
$$

\section{LINK POLYNOMIALS FROM $D(G)$ AND DIAgONALISATION OF $\sigma$}

Throughout we let $V=V_{k, \alpha}$ be a fixed, but arbitrary, irreducible $D(G)$-module and we let $P$ be the permutation operator on $V \otimes V$ considered in Section 3. In view of equation (32) we obtain the following form for the braid generator (12) and its inverse on $V \otimes V$, with respect to the orthonormal basis (30) for $V$ :

$$
\begin{aligned}
& \sigma=\sum_{s, t \in \mathcal{C}_{k}} \pi_{\alpha}^{k}\left(\tau_{a t s^{-1}}^{-1} s \tau_{t}\right)_{j i} E_{i t}^{l \ell} \otimes E_{l}^{j}: t^{-1} \\
& \sigma^{-1}=\sum_{\ell, t \in \mathcal{C}_{k}} \pi_{\alpha}^{k}\left(\tau_{,-1}^{-1} s^{-1} \tau_{t}\right)_{j i} E_{l}^{j, \iota^{-1}} \otimes E_{i t}^{l \iota}
\end{aligned}
$$


In the case $\alpha=\alpha_{0}$ corresponds to a one-dimensional representation of $Z_{k}$, these expressions simplify to

$$
\begin{aligned}
& \sigma=\sum_{,, t \in \mathcal{C}_{k}} \chi_{\alpha_{0}}^{k}\left(\tau_{s t,-1}^{-1} s \tau_{t}\right) E_{t}^{s} \otimes E_{s}^{s t s^{-1}}
\end{aligned}
$$

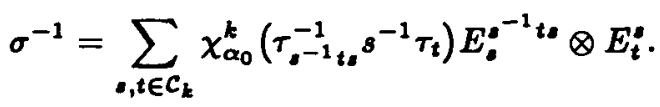

In particular when $\alpha=\iota_{0}$, corresponding to the identity representation of $Z_{k}$, we obtain [see equation (33)]

$$
\begin{aligned}
& \sigma=\sum_{s, t \in \mathcal{C}_{k}} E_{t}^{a} \otimes E_{s}^{s t s^{-1}} \\
& \sigma^{-1}=\sum_{s, t \in \mathcal{C}_{k}} E_{e^{-1} t \rho} \otimes E_{t}^{a} .
\end{aligned}
$$

The above representation of the braid generator $\sigma$, as we have seen, gives rise to a representation of the braid group $B_{n}$ on $V^{\otimes n}$. We may now proceed to define a Markov trace and corresponding link polynomial as described in Section 3. Throughout, we follow the convention of Section 3, and identify the elements of $B_{n}$, for simplicity, with their matrix representatives on $V^{\otimes n}$.

The element $u$ and its inverse of equations (9), in this case, are given by

$$
\begin{aligned}
u & =\sum_{g \in G}\left(g^{-1}\right)^{*} g=\sum_{g \in G} g\left(g^{-1}\right)^{*} \\
u^{-1} & =\sum_{g \in G} g^{*} g=\sum_{g \in G} g g^{*}
\end{aligned}
$$

which can be shown directly to satisfy equations (10). Moreover $u, u^{-1}$ are now central elements: we denote their eigenvalues on $V=V_{k, \alpha}$ by $\left\langle u^{ \pm 1}\right\rangle_{k, \alpha}$ respectively. They are related to the eigenvalues

$$
\left\langle g_{k}^{ \pm 1}\right\rangle_{\alpha}=\chi_{\alpha}^{k}\left(g_{k}^{ \pm 1}\right) / d_{\alpha}^{k}
$$

of the $Z_{k}$-invariants $g_{k}^{ \pm 1}$ respectively on the irreducible $A_{k}$-module $V_{\alpha}^{k}$, by the following lemma.

LEMMA 5.1.

$$
\left\langle u^{ \pm 1}\right\rangle_{k, \alpha}=\left[\left\langle g_{k}\right\rangle_{\alpha}\right]^{\mp 1}
$$

Proof: Observe that

$$
t r \pi_{k, \alpha}(u)=\langle u\rangle_{k, \alpha} d[k, \alpha]
$$


On the other hand, from equation (39), we have

$$
\begin{aligned}
\operatorname{tr} \pi_{k, \alpha}(u) & =\sum_{g \in G} \chi_{k, \alpha}\left(g^{*} g^{-1}\right) \\
& =\sum_{s \in \mathcal{C}_{k}} \chi_{k, \alpha}\left(s^{*} s^{-1}\right) \\
& =\sum_{s \in \mathcal{C}_{k}} \chi_{\alpha}^{k}\left(\tau_{s}^{-1} s^{-1} \tau_{\lrcorner}\right) \\
& =\left|\mathcal{C}_{k}\right| \chi_{\alpha}^{k}\left(g_{k}^{-1}\right)=d[k, \alpha] /\left\langle g_{k}\right\rangle_{\alpha}
\end{aligned}
$$

from which the result follows.

Since $u$ reduces to a scalar multiple $\langle u\rangle_{k, \alpha}=\left\langle g_{k}\right\rangle_{\alpha}^{-1}$ of the identity on $V=V_{k, \alpha}$, the $u$-dimension of equation (11) reduces to

$$
d_{u}[k, \alpha]=\langle u\rangle_{k, \alpha} d[k, \alpha]
$$

The eigenvalues $\gamma_{k, \alpha}, \bar{\gamma}_{k, \alpha}$ respectively of the invariants (14) are now given explicitly by:

LEMMA 5.2 .

$$
\gamma_{k, \alpha}=1, \quad \bar{\gamma}_{k, \alpha}=\left\langle g_{k}\right\rangle_{\alpha}^{-2}
$$

Proof: We have

$$
\begin{aligned}
\operatorname{tr}_{V \otimes V}\left[(u \otimes u) \sigma^{ \pm 1}\right] & =\langle u\rangle_{k, \alpha}^{2} \operatorname{tr} V \otimes V\left[P \sum_{g \in G} g \otimes\left(g^{ \pm 1}\right)^{*}\right] \\
& =\langle u\rangle_{k, \alpha}^{2} \operatorname{tr} \pi_{k, \alpha}\left[\sum_{g \in G} g\left(g^{ \pm 1}\right)^{*}\right] \\
& =\langle u\rangle_{k, \alpha}^{2} t r \pi_{k, \alpha}\left(u^{\mp 1}\right) \\
& =\langle u\rangle_{k, \alpha}^{2}\langle u\rangle_{k, \alpha}^{\mp 1} d[k, \alpha]
\end{aligned}
$$

In view of equation (15), we thus have

$$
\begin{aligned}
& \gamma_{k, \alpha}=\operatorname{tr}_{V \otimes V}[(u \otimes u) \sigma] / d_{u}[k, \alpha]=1 \\
& \bar{\gamma}_{k, \alpha}=\operatorname{tr}_{V \otimes V}\left[(u \otimes u) \sigma^{-1}\right] / d_{u}[k, \alpha]=\langle u\rangle_{k, \alpha}^{2}
\end{aligned}
$$

which is sufficient to prove the result.

For the case at hand, Theorem 3.2 thus reduces to (notation as before) 
THEOREM 5.1 .

$$
\varphi(\theta)=\operatorname{tr}(\theta) /(d[k, \alpha])^{n}, \quad \theta \in B_{n}
$$

satisfies the defining relations (2) of a Markov trace with

$$
z=\left\langle g_{k}\right\rangle / d[k, \alpha], \quad \bar{z}=\left\langle g_{k}^{-1}\right\rangle_{\alpha} / d[k, \alpha] .
$$

The link polynomial of Theorem 3.3 now becomes

$$
L(\widehat{\theta})=(d[k, \alpha])^{n-1}\left\langle g_{k}\right\rangle_{\alpha}^{-e(\theta)} \varphi(\theta), \quad \theta \in B_{n}
$$

which enables families of link polynomials to be constructed corresponding to each irreducible $D(G)$-module $V_{k, \alpha}$. Such link polynomials may be directly evaluated with the help of the characters of $D(G)$.

We now demonstrate, for each irreducible $D(G)$ module $V=V_{k, \alpha}$, that $\sigma$ may be diagonalised on $V \otimes V$ and obtain an analogue of the Reshetikhin form of the braid generator, obtained previously for quantum groups $[18,9]$. This diagonal form of the braid generator $\sigma$ is well known $[18,21,9]$ to be invaluable for the explicit construction of link polynomials and for the determination of their generalised Skein relations, as seen below.

The important point is that $\sigma$, given by equation (12), satisfies

$$
\begin{aligned}
\sigma^{\dagger} & =(P R)^{\dagger} \\
& =R^{\dagger} P \\
& =R^{-1} P=\sigma^{-1}
\end{aligned}
$$

and thus is a unitary operator: this is a direct consequence of the unitarity (29) of the $R$-matrix (28). In particular $\sigma$ is diagonalisable on $V \otimes V$ with eigenvalues lying on the unit circle in the complex plane: in fact it turns out that the eigenvalues of $\sigma$ are all roots of unity. From the point of view of the braid group, the matrix representation (16) thus gives rise to a unitary representation of $B_{n}$.

Since $\sigma$ and its powers commute with the action of $D(G)$ on $V \otimes V$, we may decompose the tensor product space into irreducible $D(G)$-modules on which $\sigma$ reduces to a scalar multiple of the identity. To determine the eigenvalues of $\sigma$, suppose $V_{l, \beta} \subseteq$ $V \otimes V$ is an irreducible $D(G)$ module on which $\sigma$ takes the eigenvalue $\langle\sigma\rangle_{l, \beta}$. Then we have, from equations $(10,18)$

$$
\begin{aligned}
\langle\sigma\rangle_{l, \beta}^{2} & =\left\langle\sigma^{2}\right\rangle_{l, \beta} \\
& =\left\langle R^{T} R\right\rangle_{l, \beta} \\
& =\left\langle(u \otimes u) \bar{\triangle}\left(u^{-1}\right)\right\rangle_{l, \beta} \\
& =\langle u\rangle_{k, \alpha}^{2} /\langle u\rangle_{l, \beta}=\left\langle g_{l}\right\rangle_{\beta} /\left\langle g_{k}\right\rangle_{k}^{2} .
\end{aligned}
$$


Thus the allowed eigenvalues of $\sigma$ on $V \otimes V$ are given by

$$
\pm\left\langle g_{l}\right\rangle_{\beta}^{1 / 2} /\left\langle g_{k}\right\rangle_{\alpha}
$$

where we take that square root in the right hand side of the complex plane (including the positive, but excluding the negative, imaginary axis). Following the quantum group case $[18,9]$, we refer to the phase \pm 1 occurring in equation (42) as the parity of the eigenvalue.

We let $W_{ \pm}$be the submodules of $V \otimes V$ spanned by eigenvectors with parity \pm 1 respectively: since $\sigma$ is diagonalisable we have the decomposition

$$
V \otimes V=W_{+} \oplus W_{-}
$$

We let $P[ \pm]$ be the projection operators onto the submodules $W_{ \pm}$respectively: since $\sigma$ is a $D(G)$-invariant, these projections commute with the co-product action of $D(G)$ on the tensor product module. We write the decomposition of $W_{ \pm}$into irreducible submodules according to

$$
W_{ \pm}=\bigoplus_{l, \beta} m_{l, \beta}^{ \pm} V_{l, \beta}
$$

where $m_{l, \beta}^{ \pm}$is the multiplicity of $V_{l, \beta}$ in $W_{ \pm}$respectively. We clearly have

$$
m_{l, \beta}^{+}+m_{l, \beta}^{-}=m_{l, \beta}
$$

where $m_{l, \beta}$ is the multiplicity of $V_{l, \beta}$ in $V_{k, \alpha} \otimes V_{k, \alpha}$ as given in Lemma 4.2.

We let

$$
P_{l, \beta}^{ \pm}=P[ \pm] \bar{\Delta}\left(E_{l, \beta}\right)
$$

be the projection operators onto the primary submodules occurring in the decomposition (43). In view of the remarks above, we arrive at the following spectral decomposition for $\sigma$ and its powers:

THEOREM 5.2.

$$
\sigma^{m}=\left\langle g_{k}\right\rangle_{\alpha}^{-m} \sum_{l, \beta}\left\langle g_{l}\right\rangle_{\beta}^{m / 2}\left(P_{l, \beta}^{+}+(-1)^{m} P_{l, \beta}^{-}\right), \quad m \in \mathbb{Z} .
$$

COROLlary .

$$
\sigma^{2 m}=\left\langle g_{k}\right\rangle_{\alpha}^{-2 m} \sum_{l, \beta}\left\langle g_{l}\right\rangle_{\beta}^{m} \bar{\Delta}\left(E_{l, \beta}\right), \quad m \in \mathbb{Z}
$$


Since $\sigma$ and its powers $\sigma^{m}$ are $D(G)$-invariants acting on $V \otimes V$ it follows, from Theorem 3.1 and the invariance of $u$, that the operators

$$
C_{m}=\tau_{2}\left(\sigma^{m}\right)
$$

are $D(G)$-invariants acting on $V=V_{k, \alpha}$ : here, as before, $\tau_{2}$ denotes the partial trace over the second factor of the tensor product space. Their eigenvalues $\left\langle C_{m}\right\rangle_{k, \alpha}$ are given by

THEOREM 5.3.

$$
\left\langle C_{m}\right\rangle_{k, \alpha}=\left\langle g_{k}\right\rangle_{\alpha}^{-m} \sum_{l, \beta}\left(g_{l}\right\rangle_{\beta}^{m / 2}\left(m_{l, \beta}^{+}+(-1)^{m} m_{l, \beta}^{-}\right) \frac{d[l, \beta]}{d[k, \alpha]}
$$

Proof: In view of Theorem 5.2, we have

$$
\begin{aligned}
\left\langle C_{m}\right\rangle_{k, \alpha} d[k, \alpha] & =\operatorname{tr}_{V \otimes V}\left(\sigma^{m}\right) \\
& =\left\langle g_{k}\right\rangle_{\alpha}^{-m} \sum_{l, \beta}\left\langle g_{l}\right\rangle_{\beta}^{m / 2} \operatorname{tr}_{V \otimes V}\left(P_{l, \beta}^{+}+(-1)^{m} P_{l, \beta}^{-}\right) .
\end{aligned}
$$

The result is then seen to follow from

$$
\operatorname{tr} V \otimes V_{V}\left[P_{l, \beta}^{ \pm}\right]=m_{l, \beta}^{ \pm} d[l, \beta] .
$$

The multiplicities $m_{l, \beta}^{ \pm}$(possibly zero) may be determined explicitly with the help of equation (44) and the following result:

LEMMA 5.3 .

$$
m_{l, \beta}^{+}-m_{l, \beta}^{-}=\frac{\left\langle g_{k}\right\rangle_{\alpha}}{\left\langle g_{l}\right\rangle_{\beta}^{1 / 2}} \mu_{l, \beta}
$$

where

$$
\mu_{l, \beta}=\frac{1}{\left|Z_{k}\right|} \sum_{g \in G} \chi_{l, \beta}\left[g^{-1}\left(g_{k} g g_{k} g^{-1}\right)^{*}\right] \chi_{k, \alpha}\left(g g_{k} g g_{k}^{*}\right)
$$

Proof: In view of equation (36) we have

$$
\begin{aligned}
\sigma \bar{\Delta}\left(E_{l, \beta}\right) & =\frac{d[l, \beta]}{|G|} \sum_{s \in \mathcal{C}_{l}} \sum_{g} \chi_{l, \beta}\left(g^{-1} s^{*}\right) \sigma \bar{\triangle}\left(s^{*} g\right) \\
& =\frac{d[l, \beta]}{|G|} \sum_{t \in G} \sum_{s \in \mathcal{C}_{l}} \sum_{g} \chi_{l, \beta}\left(g^{-1} s^{*}\right) P\left[t\left(t^{-1} s\right)^{*} g \otimes t^{*} g\right]
\end{aligned}
$$


from which we obtain

$$
\begin{gathered}
\operatorname{tr}_{V \otimes V}\left[\sigma \bar{\triangle}\left(E_{l, \beta}\right)\right]=\frac{d[l, \beta]}{|G|} \sum_{\substack{t \in \mathcal{C}_{k} \\
s \in \mathcal{C}_{l}}} \sum_{g} \chi_{l, \beta}\left(g^{-1} s^{*}\right) \chi_{k, \alpha}\left(t\left(t^{-1} s\right)^{*} g t^{*} g\right) \\
=\frac{d[l, \beta]}{|G|} \sum_{t \in \mathcal{C}_{k}} \sum_{g} \chi_{l, \beta}\left(g^{-1}\left(t g t g^{-1}\right)^{*}\right) \chi_{k, \alpha}\left(g t g t^{*}\right) .
\end{gathered}
$$

On the other hand, from Theorem 5.2, we have

$$
\operatorname{tr} V \otimes V_{\sigma}\left[\sigma \bar{\triangle}\left(E_{l, \beta}\right)\right]=\frac{\left\langle g_{l}\right\rangle_{\beta}^{1 / 2}}{\left\langle g_{k}\right\rangle_{\alpha}}\left(m_{l, \beta}^{+}-m_{l, \beta}^{-}\right) d[l, \beta] .
$$

It follows that

$$
\left(m_{l, \beta}^{+}-m_{l, \beta}^{-}\right) \frac{\left\langle g_{l}\right\rangle_{\beta}^{1 / 2}}{\left\langle g_{k}\right\rangle_{\alpha}}=\mu_{l, \beta}
$$

with

$$
\mu_{l, \beta}=\frac{1}{|G|} \sum_{t \in \mathcal{C}_{k}} \sum_{g} \chi_{l, \beta}\left[g^{-1}\left(t g g^{-1}\right)^{*}\right] \chi_{k, \alpha}\left(g t g t^{*}\right) .
$$

Finally using $t=\tau_{t}^{-1} g_{k} \tau_{t}$, together with the invariance properties of characters, we arrive at

$$
\begin{aligned}
\mu_{l, \beta} & =\frac{1}{|G|} \sum_{t \in \mathcal{C}_{k}} \sum_{g} \chi_{l, \beta}\left[g^{-1}\left(g_{k} g g_{k} g^{-1}\right)^{*}\right] \chi_{k, \alpha}\left(g g_{k} g g_{k}^{*}\right) \\
& =\frac{\left|\mathcal{C}_{k}\right|}{|G|} \sum_{g} \chi_{l, \beta}\left[g^{-1}\left(g_{k} g g_{k} g^{-1}\right)^{*}\right] \chi_{k, \alpha}\left(g g_{k} g g_{k}^{*}\right)
\end{aligned}
$$

which is sufficient to prove the result.

REMARKS.

(1) In the case $m_{l, \beta}=1, \mu_{l, \beta}$ as above in fact gives the eigenvalue of $\sigma$ on $V_{l, \beta} \subseteq V_{k, \alpha} \otimes V_{k, \alpha}$.

(2) From Theorem 5.3, the eigenvalues of the even and odd order invariants (45), may thus be expressed

$$
\begin{aligned}
\left\langle C_{2 m}\right\rangle_{k, \alpha} & =\left\langle g_{k}\right\rangle_{\alpha}^{-2 m} \sum_{l, \beta}\left\langle g_{l}\right\rangle_{\beta}^{m} m_{l, \beta} \frac{d[l, \beta]}{d[k, \alpha]} \\
\left\langle C_{2 m+1}\right\rangle_{k, \alpha} & =\left\langle g_{k}\right\rangle_{\alpha}^{-2 m} \sum_{l, \beta}\left\langle g_{l}\right\rangle_{\beta}^{m} \mu_{l, \beta} \frac{d[l, \beta]}{d[k, \alpha]}
\end{aligned}
$$


with $\mu_{l, \beta}$ as above and $m_{l, \beta}$ as in Lemma 4.2.

We note that Theorem 5.2 implies the following identity satisfied by the braid generator $\sigma$ :

$$
\prod_{l, \beta}^{\prime}\left(\sigma-\left\langle g_{l}\right\rangle_{\beta}^{1 / 2} /\left\langle g_{k}\right\rangle_{\alpha}\right)\left(\sigma+\left\langle g_{l}\right\rangle_{\beta}^{1 / 2} /\left\langle g_{k}\right\rangle_{\alpha}\right)=0 .
$$

Here the prime signifies that we retain only those factors corresponding to $m_{l, \beta}^{ \pm} \neq 0$. Expanding the left-hand side into powers of $\sigma$ we arrive at the identity

$$
\sigma^{m}-\sum_{l=0}^{m-1} a_{l} \sigma^{l}=0
$$

which leads to the generalised Skein relations for the link polynomials (40). For example, with

$$
\theta=\theta_{1} \sigma_{i}^{m} \theta_{2}, \quad \theta_{1}, \theta_{2} \in B_{n}
$$

we have

$$
L(\widehat{\theta})=\sum_{l=0}^{m-1} a_{l} L\left(\widehat{\theta_{1} \sigma_{i}^{l} \theta_{2}}\right) .
$$

Finally, to illustrate the utility of this formalism, we now obtain a general formula for $L(\widehat{\theta})$, when $\theta$ is a braid of the following form:

$$
\theta=\left(\sigma_{i_{1}}\right)^{m_{1}}\left(\sigma_{i_{2}}\right)^{m_{2}} \ldots \ldots\left(\sigma_{i_{n-1}}\right)^{m_{n-1}}, \quad m_{i} \in \mathbb{Z}
$$

where $\left(i_{1}, \ldots, i_{n-1}\right)$ is an arbitrary permutation of $(1,2, \ldots, n-1)$. From Theorem 5.3, together with the definition $(40)$ of $L(\widehat{\theta})$, it is straightforward to show that

$$
\begin{aligned}
& L(\widehat{\theta})=\left\langle g_{k}\right\rangle_{\alpha}^{-\sum_{i=1}^{n-1} m_{i}} \prod_{i=1}^{n-1}\left\langle C_{m_{i}}\right\rangle_{k, \alpha} \\
& =(d[k, \alpha])^{1-n}\left\langle g_{k}\right\rangle_{\alpha}^{-2} \sum_{i=1}^{n-1} m_{i} \prod_{i=1}^{n-1}\left\{\sum_{l, \beta}\left\langle g_{l}\right\rangle_{\beta}^{1 / 2 m_{i}}\left(m_{l, \beta}^{+}+(-1)^{m_{i}} m_{l, \beta}^{-}\right) d[l, \beta]\right\} .
\end{aligned}
$$

For example, for the trefoil knot $\theta_{t}$ corresponding to

$$
\theta_{t}=\sigma_{1}^{3} \in B_{2}
$$

we obtain

$$
\begin{aligned}
L\left(\widehat{\theta}_{t}\right) & =\left\langle g_{k}\right\rangle_{\alpha}^{-6} \sum_{l, \beta}\left\langle g_{l}\right\rangle_{\beta}^{3 / 2}\left(m_{l, \beta}^{+}-m_{l, \beta}^{-}\right) \frac{d[l, \beta]}{d[k, \alpha]} \\
& =\left\langle g_{k}\right\rangle_{\alpha}^{-5} \sum_{l, \beta}\left\langle g_{l}\right\rangle_{\beta} \mu_{l, \beta} \frac{d[l, \beta]}{d[k, \alpha]}
\end{aligned}
$$


In what follows we shall apply the above proceedure to obtain link polynomials arising from a certain irreducible unitary representation of $D\left(S_{N}\right), S_{N}$ being the symmetric group.

\section{Link Polynomials from $D\left(S_{N}\right), N>4$}

Let $\mathcal{C}_{2} \subseteq S_{N}$ be the conjugacy class of transpositions with representative $g_{2}=$ $(N-1 N)$. The centraliser subgroup $Z_{2}$ of $g_{2}$ is given by

$$
Z_{2}=S_{N-2} \times S_{2}
$$

with $S_{2}=\left\{1, g_{2}\right\}$ and $S_{N-2}$ the natural subgroup. Note that

$$
\left|\mathcal{C}_{2}\right|=\frac{1}{2} N(N-1)=\left|S_{N}\right| /\left|Z_{2}\right|
$$

as required. We let $V_{1}^{2}$ denote the one dimensional module corresponding to the identity representation of $Z_{2}$. Here we diagonalise $\sigma$ on the tensor product module $V_{2,1} \otimes V_{2,1}$ where $V_{2,1}$ denotes the irreducible $D\left(S_{N}\right)$ module induced by $V_{1}^{2}$ : the dimension of this module is given by

$$
d[2,1]=\left|\mathcal{C}_{2}\right|=\frac{1}{2} N(N-1)
$$

In view of the remark proceeding Lemma 4.2, it is easily seen that the only $D\left(S_{N}\right)$ modules occurring in the above tensor product are those of the form $V_{k, \alpha}$ where $\mathcal{C}_{k}$ is a conjugacy class occurring in

$$
\mathcal{C}_{2} \cdot \mathcal{C}_{2}=\mathcal{C}_{1} \cup \mathcal{C}_{3} \cup \mathcal{C}_{5}
$$

Here $\mathcal{C}_{1}=\{1\}, \mathcal{C}_{3}$ is the class of 3 cycles, and $\mathcal{C}_{5}$ is the class of $2 \times 2$ cycles: note that

$$
\left|\mathcal{C}_{1}\right|=1, \quad\left|\mathcal{C}_{3}\right|=2\left(\begin{array}{c}
N \\
3
\end{array}\right), \quad\left|\mathcal{C}_{5}\right|=3\left(\begin{array}{c}
N \\
4
\end{array}\right)
$$

where, as usual,

$$
\left(\begin{array}{l}
N \\
m
\end{array}\right)=\frac{N !}{m !(N-m) !}, \quad 0 \leqslant m \leqslant N .
$$

As coset representatives we choose

$$
g_{1}=1, \quad g_{3}=(N-2 N-1 N), \quad g_{5}=(N-1 N)(N-2 N-3)
$$

respectively. The corresponding centraliser subgroups in this case are

$$
Z_{1}=S_{N}, \quad Z_{3}=S_{N-3} \times C_{3}
$$


where $S_{N-3}$ is the obvious subgroup with $C_{3}=\left\{1, g_{3}, g_{3}^{-1}\right\}$ the cyclic group of order 3 , and

$$
Z_{5}=\left(S_{N-4} \times S_{2} \times S_{2}\right) \text { (S) } T .
$$

Here $S_{N-4}$ is the natural subgroup,

$$
T=\{(N-1 N-3)(N-2 N),(N-2 N-1)(N-3 N)\},
$$

and the two $S_{2}$ groups are given respectively by

$$
S_{2}=\{1,(N-3 N-2)\}, S_{2}=\{1,(N-1 N)\} .
$$

It is worth noting that these groups have orders

$$
\left|Z_{1}\right|=N !,\left|Z_{3}\right|=3(N-3) !,\left|Z_{5}\right|=8(N-4) !
$$

in agreement with

$$
\left|Z_{k}\right|=|G| /\left|\mathcal{C}_{k}\right|
$$

For the problem at hand we are only concerned with three irreducible $Z_{1}$ modules, denoted $V_{\alpha}^{1}(1 \leqslant \alpha \leqslant 3)$, which give rise to the irreducible representations of $Z_{1}=S_{N}$ with Young diagrams $[N],[N-11],[N-22]$ respectively: these correspond to the identity representation and the irreducible representations of dimensions $N-1$ and $N(N-3) / 2$ respectively. The corresponding irreducible $D\left(S_{N}\right)$ modules $V_{1, \alpha}(1 \leqslant \alpha \leqslant 3)$ have the same dimensions so that

$$
d[1,1]=1, \quad d[1,2]=N-1, \quad d[1,3]=\frac{1}{2} N(N-3) .
$$

For the centraliser subgroup $Z_{3} \cong S_{N-3} \times C_{3}$ we let $V_{\alpha}^{3}(1 \leqslant \alpha \leqslant 3)$ denote the irreducible one dimensional modules corresponding to the identity representation of $S_{N-3}$ and the three irreducible representations of the cyclic group $C_{3}$ : their respective characters are thus determined by

$$
\chi_{1}^{3}\left(g_{3}\right)=1, \quad \chi_{2}^{3}\left(g_{3}\right)=\omega, \quad \chi_{3}^{3}\left(g_{3}\right)=\bar{\omega}
$$

where $\omega=e^{i 2 \pi / 3}$. Finally we let $V_{\alpha}^{5}(\alpha=1,2)$ be the one dimensional modules corresponding to the identity representation of $Z_{5}$ and the one dimensional representation with character determined by

$$
\chi_{2}^{5}(g)= \begin{cases}1, & g \in S_{N-4} \times S_{2} \times S_{2} \\ -1, & g \in T\end{cases}
$$


respectively. The corresponding irreducible induced $D\left(S_{N}\right)$ modules $V_{3, \alpha}(1 \leqslant \alpha \leqslant 3)$, $V_{5, \alpha}(\alpha=1,2)$ have dimensions

$$
\begin{aligned}
& d[3, \alpha]=\left|\mathcal{C}_{3}\right|=\frac{1}{3} N(N-1)(N-2), \quad 1 \leqslant \alpha \leqslant 3 \\
& d[5, \alpha]=\left|\mathcal{C}_{5}\right|=\frac{1}{8} N(N-1)(N-2)(N-3), \quad \alpha=1,2 .
\end{aligned}
$$

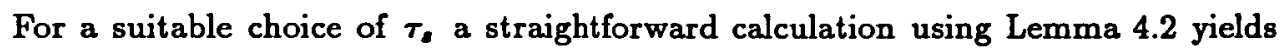
the tensor product decomposition

$$
V_{2,1} \otimes V_{2,1}=V_{1,1} \oplus V_{1,2} \oplus V_{1,3} \oplus V_{3,1} \oplus V_{3,2} \oplus V_{3,3} \oplus V_{5,1} \oplus V_{5,2}
$$

Since this decomposition is multiplicity-free, the eigenvalues of $\sigma$ on the irreducible modules above are given the corresponding $\mu$-values of equation (46) which are listed below:

$$
\begin{aligned}
& \mu_{1,1}=\mu_{1,2}=\mu_{1,3}=\mu_{3,1}=\mu_{5,1}=1 \\
& \mu_{3,2}=\bar{\omega}, \quad \mu_{3,3}=\omega, \quad \mu_{5,2}=-1 .
\end{aligned}
$$

We thus arrive at the following spectral decomposition in terms of the (central) projections $\bar{\Delta}\left(E_{k, \alpha}\right)$ acting on the tensor product space:

$$
\begin{aligned}
\sigma= & \bar{\triangle}\left(E_{1,1}\right)+\bar{\Delta}\left(E_{1,2}\right)+\bar{\Delta}\left(E_{1,3}\right)+\bar{\Delta}\left(E_{3,1}\right) \\
& +\bar{\omega} \bar{\Delta}\left(E_{3,2}\right)+\omega \bar{\Delta}\left(E_{3,3}\right)+\bar{\Delta}\left(E_{5,1}\right)-\bar{\Delta}\left(E_{5,2}\right) .
\end{aligned}
$$

In this case the eigenvalues of the invariants (45) are given by

$$
\begin{aligned}
\left\langle C_{m}\right\rangle_{2,1}= & (d[1,1])^{-1}(d[1,1]+d[1,2]+d[1,3]+d[3,1] \\
& \left.+\bar{\omega}^{m} d[3,2]+\omega^{m} d[3,3]+d[5,1]+(-1)^{m} d[5,2]\right)
\end{aligned}
$$

which, using the dimensions given above, simplifies to

$$
\left\langle C_{m}\right\rangle_{2,1}=1+\frac{2}{3}(N-2)\left[1+2 \cos \left(\frac{2 m \pi}{3}\right)\right]+\frac{1}{4}(N-2)(N-3)\left[1+(-1)^{m}\right] .
$$

Substituting into equation (48) then gives the link polynomial

$$
L(\widehat{\theta})=\prod_{i=1}^{n-1}\left\{1+\frac{2}{3}(N-2)\left[1+2 \cos \left(\frac{2 m_{i} \pi}{3}\right)\right]+\frac{1}{4}(N-2)(N-3)\left[1+(-1)^{m_{i}}\right]\right\}
$$

for $\theta$ a braid of the general form (47).

In this way we obtain an infinite family of link polynomials corresponding to each integer $N>4$. Replacing $N$ by a parameter $q$, it is possible that the above in fact determines a one-variable link polynomial. It would be of interest to examine in further detail such link polynomials arising from more general representations of $D\left(S_{N}\right)$. 
REMARK. It is still an open problem as to whether the $R$-matrices $(32,33)$ can be Baxterised to yield solutions of the parameter-dependent Yang-Baxter equation [3, 14, 11) occurring in statistical mechanics. Such parameter-dependent solutions would then define exactly solvable models associated with certain induced representations of a finite group. Further work along these lines is now in progress.

\section{REFERENCES}

[1] E. Abe, Hopf algebras (Cambridge University Press, Cambridge, 1980).

[2] J.W. Alexander, 'Topological invariants of knots and links', Trans. Amer. Math. Soc. 30 (1928), 275-306.

[3] R.J. Baxter, Exactly solved models in statistical mechanics (Academic Press, New York, 1982).

[4] V.G. Drinfeld, 'Quantum groups', in Proc. ICM Berkeley 1 (Amer. Math. Soc., Providence, R.I., 1987), pp. 798-820.

[5] V.G. Drinfeld, 'Quesi cocommutative Hopf algebras', Algebra and Analysis 2 (1988), $30-46$.

[6] L.D. Faddeev, N. Yu. Reshetikhin and L.A. Takhtajan, 'Quantum groups', Algebra and Analysis 1 (1988), 129-139.

[7] M.D. Gould, R.B. Zhang and A.J. Bracken, 'Generalized Gelfand invariants and characteristic identities for Quantum groups', J. Math. Phys. 32 (1991), 2298-2303.

[8] M.D. Gould, 'Quantum-double finite group algebras and their representations' (to appear), Univ. of Queensland preprint.

[9] M.D. Gould, 'Quantum groups and diagonalization of the Braid generator', Lett. Math. Phys. 24 (1992), 183-196.

[10] M.D. Gould and J. Links, 'Casimir invariants for Hopf algebras', Rep. Math. Phys. 31 (1992), 91-111.

[11] M. Jimbo., 'A $q$-difference analogue of $U(g)$ and the Yang-Baxter equation', Lett. Math. Phys. 10 (1985), 63-69.

[12] V.F.R. Jones, 'A polynomial invariant for links via von Neumann algebras', Bull. Amer. Math. Soc. 12 (1985), 103-112.

[13] L.H. Kauffman, On knots, Annals of Math. Study 115 (Princeton University Press, 1981 ).

[14] P.P. Kulish and E.K. Sklyanin, 'Quantum spectral transforms method. Recent developments', in Integrable quantum field theories, Lecture notes in Physics 151 (Springer-Verlag, Berlin, Heidelberg, New York, 1982), pp. 61-119.

[15] R.G. Larson, 'Characters of Hopf algebras', J. Algebra 17 (1971), 352-368.

[16] A.A. Markov, 'Über die freie Äquivalenz geschlossener Zöpfe', Recueil Math. Moscow 1 (1935), 73-78.

[17] K. Reidemeister, Knotentheorie (Chelsea, New York, 1948).

[18] N.Yu. Reshetikhin, 'Qunatized universal enveloping algebras, the Yang-Baxter equation and invariants of Links I, II', preprints E-4-87, E-17-87, L.O.M.I. (Leningrad). 
[19] M.E. Sweedler, Hopf algebras (Benjamin, New York, 1969).

[20] V.G. Turaev, 'The Yang-Baxter equation and invariants of links', Invent. Math. 92 (1988), 527-553.

[21] R.B. Zhang, M.D. Gould and A.J. Bracken, 'Quantum group invariants and link polynomials', Commun. Math. Phys. 137 (1991), 13-27.

Department of Mathematics

The University of Queensland

Queensland 4072

Australia 